\section{Informationen zur SEPA-Umstellung}

Liebe DEGUM-Mitglieder,

wie Sie sicher den Medien entnommen haben, wird der deutsche Zahlungsverkehr auf den einheitlichen europäischen Zahlungsverkehr SEPA (Single Euro Payment Area) umgestellt.

Ihre bisher erteilten Einzugsermächtigungen werden von uns als SEPA-Lastschriftmandat weiter genutzt, Sie müssen daher keine weiteren Schritte veranlassen.

Ihre SEPA-Lastschrift-Informationen sind folgende:
Zahlungsempfänger: Deutsche Gesellschaft für Ultraschall in der Medizin e.V. - DEGUM

Gläubiger-Identifikationsnummer: DE40ZZZ00000747992

Mandatsreferenz:

Ihre Mitgliedsnummer
Den Mitgliedsbeitrag in Höhe von 100,00 $€$ (lt. Beschluß der Mitgliederversammlung vom 11.10.2013 in Stuttgart ab 01.01.2014) ziehen wir jährlich zum 15.02. mit einer SEPA-Basis-Lastschrift von den Konten der Mitglieder ein. Fällt der Fälligkeitstag auf ein Wochenende/ Feiertag, verschiebt sich der Fälligkeitstag auf den nächsten Werktag.

Mit freundlichen Grüßen

Marion Schapheer-Risse DEGUM-Geschäftsstelle 